\title{
Healthcare provision during a sporting mass gathering: A structure and process description of onsite care delivery
}

Amy N.B. Johnston ${ }^{1,2,5}$, Jasmine Wadham ${ }^{1}$, Josea Polong-Brown ${ }^{1}$, Michael Aitken ${ }^{1,2}$, Jamie Ranse ${ }^{1,2}$, Alison Hutton ${ }^{3}$, Brent Richards ${ }^{4}$, Julia Crilly ${ }^{1,2}$

\section{INTRODUCTION}

During mass gatherings such as marathons the provision of timely access to healthcare services is required for the mass gathering population as well as the local community. However, effective provision of healthcare during sporting mass gatherings is not well understood.

\section{AIM}

The aim of this study was to describe the structures and processes developed for an emergency team to operate an in-event acute healthcare facility during one of the largest mass sporting participation events in the southern hemisphere, the Gold Coast marathon.

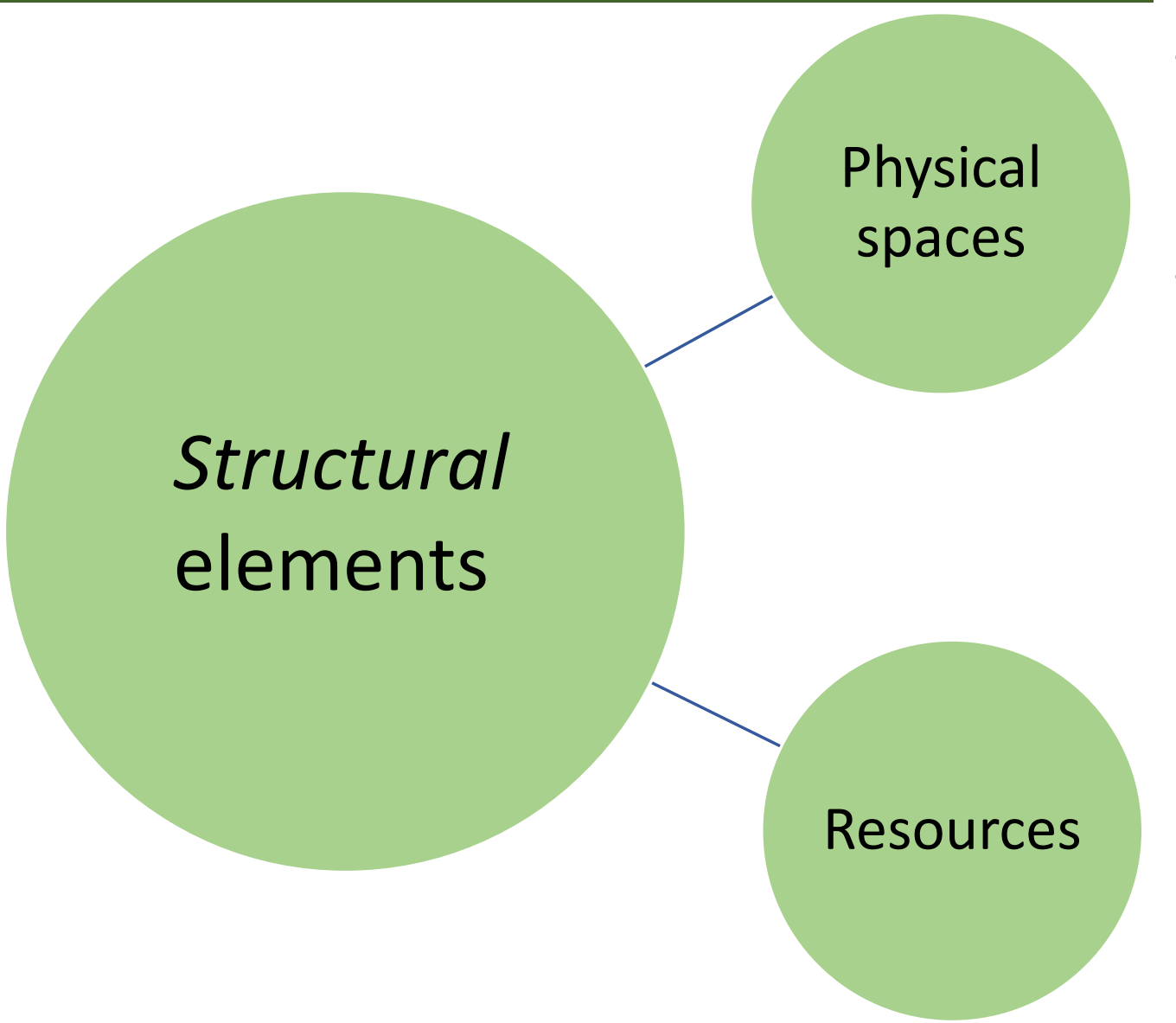

- clinical zones in the marathon health tent,

- tent access and egress points

bilingual staff,

- senior medical staff,

- equipment such as electrocardiograms

Findings

Of the 12 participants interviewed, 8 were nurses and 4 were doctors with an range of years clinical experience from 0.5-29 years and 3-25 years respectively. Structure and process elements underpinning the on-site care delivery are displayed in Figure 1 and 2, respectively.
A pragmatic qualitative methodology was used to describe the structures and processes required to operate an in-event acute healthcare facility. Content analysis from 12 semi-structured interviews with Emergency Department (ED) clinical staff working during the two-day event was undertaken in 2016. This study received HREC approval.
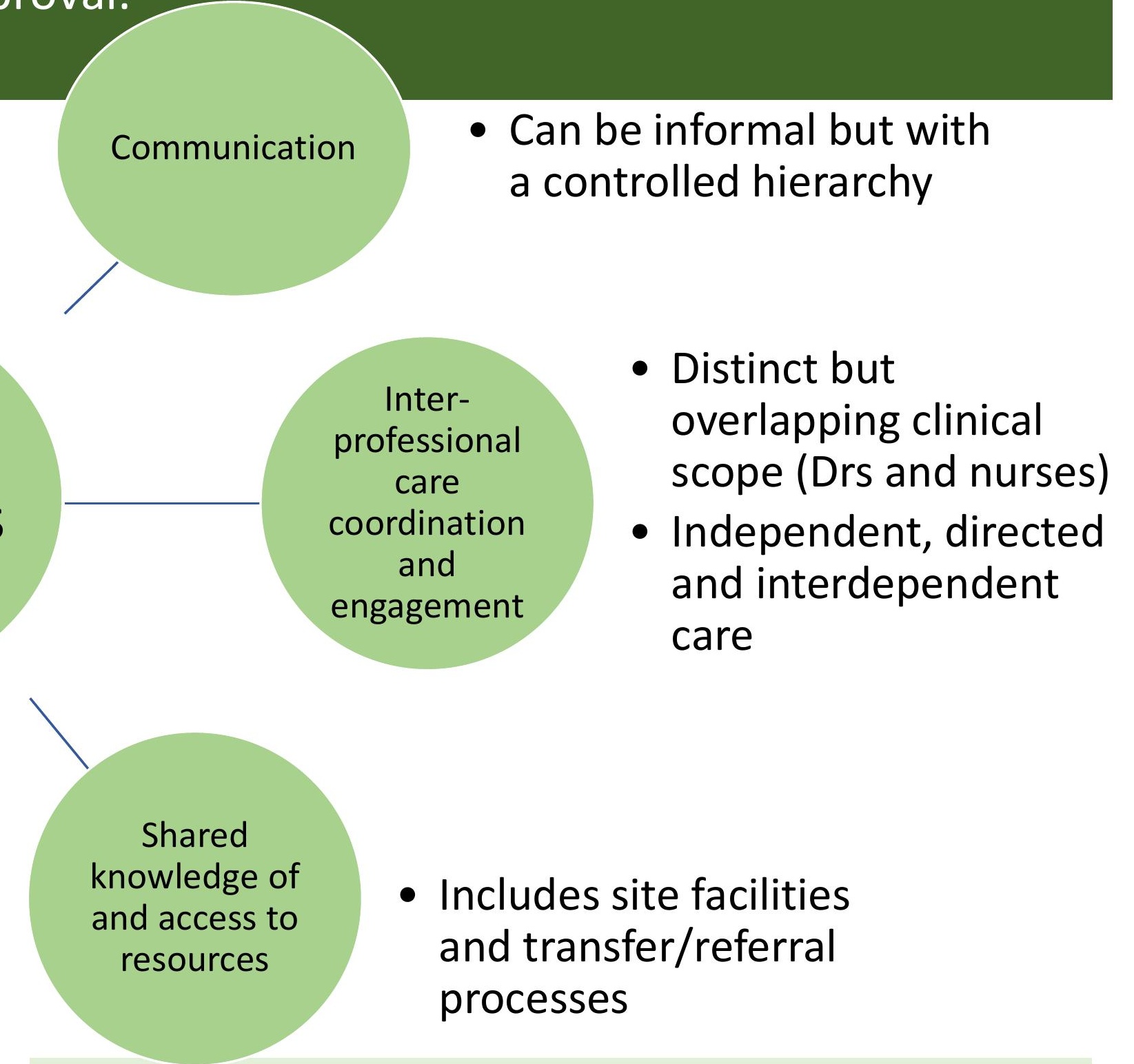

\section{DISCUSSION}

This in-event model of emergency care delivery enabled acute out-of-hospital healthcare to be delivered in a portable and transportable facility. Clinical staff reported satisfaction with their ability to provide a meaningful contribution to hospital avoidance and to the local community. With the number of sporting mass gatherings increasing, this temporary, in-event model of healthcare provision is one option for event and healthcare planners to consider.

1.Department of Emergency Medicine, Gold Coast Hospital and Health Service, D Block, LG096 1 Hospital Blvd, QLD 4215

2. Menzies Health Institute Queensland, Griffith University QLD 4222

3. School of Nursing University of Newcastle, University Dr, Callaghan NSW 2308

4. Intensive Care Unit, Gold Coast Hospital and Health Service, 1 Hospital Blvd, QLD 4215

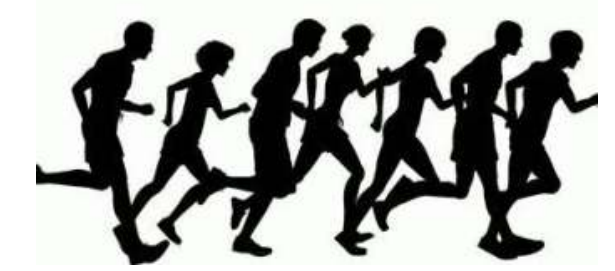

5. Now at Department of Emergency Medicine, Princess Alexandra Hospital Metro South 4102 QLD Australia and School of Nursing, Midwifery and Social Work, University of Queensland 4102 QLD Australia 